\title{
The design of solar synoptic chart for space weather forecast
}

\author{
Qiao Song ${ }^{1,2}$, Jing-Song Wang ${ }^{1}$, Xue-Shang Feng $^{2}$ and Xiao-Xin Zhang ${ }^{1}$ \\ ${ }^{1}$ Key Laboratory of Space Weather, National Center for Space Weather, \\ China Meteorological Administration, 100081, Beijing, China. \\ email: wangjs@cma.gov.cn \\ ${ }^{2}$ State Key Laboratory of Space Weather, National Space Science Center, \\ Chinese Academy of Sciences, 100190, Beijing, China.
}

\begin{abstract}
The Sun drives most events of space weather in the vicinity of the Earth. Because the activities of the Sun are complicated, a visualized chart with key objects of solar activities is needed for space weather forecast. This work investigates the key objects in research during the past forty years and surveys a variety of solar observational data. We design the solar synoptic chart (SSC) that covers the key objects of solar activities, i.e., active regions, coronal holes, filaments/prominences, flares and coronal mass ejections, and synthesizes images from different heights and temperatures of solar atmosphere. The SSC is used to analyze the condition of the Sun in March 2012 and October 2014 as examples. The result shows that the SSC is timely, comprehensive, concise and easy to understand. It has the potentiality for space weather forecast and can help in improving the public education.
\end{abstract}

Keywords. Space weather, Solar flare, Coronal mass ejection, Filament, Coronal hole

\section{Introduction}

Our modern society is deeply influenced by space weather. The Sun is the primary source of space weather in the vicinity of the Earth (Schwenn 2006). The Sun's behavior and its influence on space weather are complicated in multiple time scales. If we clarify the Sun's complex influence into a long-term background and short-term disturbances, then the average state of the Sun determines the background, and solar eruptive activities are disturbances in solar-terrestrial environment. From the viewpoint of space weather forecast, the background generally includes active regions, coronal holes, and filaments/prominences; the disturbances mainly include flares, coronal mass ejections (CMEs) and high-speed solar winds. From the perspective of solar physics, they are all driven by the magnetic fields of the Sun.

More and more institutes and organizations have begun to focus on the research and service of space weather. The National Center for Space Weather (NCSW) of China Meteorological Administration has been carrying out the prediction of space weather events for a dozen years. NCSW provides monitoring, early warning, three-day forecast, weekly report, monthly report, annual report and other space weather services, for solar flares, the sunspot number, the F107 index, geomagnetic storms, and ionospheric disturbance etc.

Solar observational satellites and ground-based telescopes have provided numerous and various data that reveal the background and disturbances of the Sun. Many previous works have made efforts to organize and visualize the immense volume of solar data, such as Solar Monitor (solarmonitor.org), Helioviewer (helioviewer.org, Stys et al. 2015), and Integrated Space Weather Analysis system (iswa.ccmc.gsfc.nasa.gov). Furthermore, new techniques of image recognition can more easily identify objects on the Sun, for 
Table 1. Briefing of Literature Investigation.

\begin{tabular}{crr}
\hline Date & Citation & Number \\
\hline $1973-1982$ & $\geqslant 50$ & 5 \\
$1983-1992$ & $\geqslant 40$ & 34 \\
$1993-2002$ & $\geqslant 30$ & 561 \\
$2003-2010$ & $\geqslant 20$ & 799 \\
$2010-2012$ & $\geqslant 10$ & 179 \\
2013 & $\geqslant 5$ & 8 \\
\hline \multicolumn{2}{c}{ Total Number: } & 1586 \\
\hline
\end{tabular}

example, active regions, coronal holes, filaments/prominences, flares, and CMEs $(\mathrm{Qu}$ et al. 2003; Henney \& Harvey 2005; Morgan et al. 2012; Hao et al. 2013; Verbeeck et al. 2013). However, there are still some things can be improved for space weather forecast. Firstly, focusing on the key objects of solar activities is a better strategy for forecasting space weather than looking after all the features on the Sun. Secondly, the magnetic fields need further attention because it is irreplaceable. Finally, because the quantitative information is useful for understanding the mechanism of solar activity (Song et al. 2013), the parameters of key objects need to be characterized.

Based on the latest data, advanced techniques and rich forecast experiences, we develop a new method, the solar synoptic chart (SSC), which is analogous to the weather chart in meteorology, to meet the need of space weather forecast and even that of the public education. In this paper, we investigate the past 40 years' researches and survey multiple solar data, to check the choice of the key objects and determine optimal data to make the SSC. Then we analyze the overall situation of solar activities on 2012 March 7 and 2014 October 23 by using the SSC as examples. In the end, we summarize this work and discuss possible improvements and applications in the future.

\section{Data and Design of SSC}

The first source of the data in this work is the research literatures in solar physics from 1973 to 2013 by the retrieve tool of Science Citation Index Expanded. We use lower limits of citations that decrease with time to reduce the quantity and to improve the quality of selected literatures. Table 1 gives the selection criteria of publication dates, lower limits of citations, and numbers of selected literatures. The literatures were searched in January 2014, and the numbers may increase with time. A total of 1586 literatures meet the criteria and the full texts of 1437 literatures are downloaded for further analysis.

The second source of the data in this work is solar observations from various instruments at different energy/frequency ranges. We investigate which objects, for example, active regions, coronal holes, and flares etc., can be detected in these observational data. This work also uses observations in white light, $\mathrm{H} \alpha$ and magnetic field from NCSW. White-light and $\mathrm{H} \alpha$ data are observed by the $15-\mathrm{cm} \mathrm{H} \alpha$ telescope of NCSW, which is located at Shidao, Shandong Province, China. Its spatial resolution is $\sim 1^{\prime \prime}$ with a $2048 \times 2048$ pixels CCD when the influence of atmospheric seeing is ignored. It can provide full-disk white-light and $\mathrm{H} \alpha$ images every three minutes. The vector magnetic field telescope of NCSW at Wenquan, Xinjiang Uygur Autonomous Region, China, provides longitudinal and vector magnetograms of the Sun. This telescope also has a $2048 \times 2048$ pixels CCD and $\sim 1^{\prime \prime}$ of spatial resolution, and records a full-disk vector magnetogram every half an hour.

Based on the literature investigation and data survey, we design the SSC method. The 
Table 2. Statistics of keywords in literature investigation.

\begin{tabular}{ccccccc}
\hline \multirow{2}{*}{$\begin{array}{c}\text { Keywords } \\
\text { of Objects }\end{array}$} & \multirow{2}{*}{$\begin{array}{c}\text { Numbers of } \\
\text { Literatures }\end{array}$} & EUV & WL & H $\alpha$ & Mag. & Tot. \\
\cline { 3 - 7 } & & & & & \\
AR & $879(62 \%)$ & $553(63 \%)$ & $230(26 \%)$ & $175(20 \%)$ & $747(85 \%)$ & $846(96 \%)$ \\
CH & $626(44 \%)$ & $353(56 \%)$ & $187(30 \%)$ & $75(12 \%)$ & $548(88 \%)$ & $602(96 \%)$ \\
FL/PR & $662(47 \%)$ & $403(61 \%)$ & $197(30 \%)$ & $164(25 \%)$ & $584(88 \%)$ & $646(98 \%)$ \\
Flare & $856(61 \%)$ & $527(62 \%)$ & $217(25 \%)$ & $173(20 \%)$ & $746(87 \%)$ & $828(97 \%)$ \\
CME & $872(62 \%)$ & $465(53 \%)$ & $256(29 \%)$ & $158(18 \%)$ & $773(89 \%)$ & $836(96 \%)$ \\
\hline
\end{tabular}

importance of an object in research can be estimated from the frequency of its searching keyword in literatures. A reference management software, EndNote, is used for searching keywords in titles, abstracts, and full texts of the literatures to check the importance. The number and percentage of literatures that contain the keyword "data" are 1408 and $89 \%$, respectively. Table 2 shows the statistics of keywords in the investigation for the 1408 literatures. The first column gives keywords of the five objects, active region $(\mathrm{AR})$, coronal hole $(\mathrm{CH})$, filament/prominence (FL/PR), flare, and CME. The second column lists the number of literatures that contains each keyword, and its percentage of the 1408 literatures is given in parentheses. Note that because a literature may contain more than one keyword, the total percentage is greater than $100 \%$. The other columns are the numbers of literatures that contain the four keywords of data, extreme ultraviolet (EUV), white light (WL), $\mathrm{H} \alpha$, magnetic field (Mag.), and the total number of literatures that contain at least one of the four keywords (Tot.). Their percentages are given in parentheses.

As shown in Table 2, EUV data appear in more than half of the literatures, which indicates that they are important for all the five key objects. The magnetic field is even more critical, because the keyword occurs more than $85 \%$ in every object. The whitelight data are also basic observation and their percentages are 25\%-30\%. H $\alpha$ data have a percentage of $25 \%$ for filaments/prominences, which is higher than other four objects. Moreover, for every object, the total percentage (>96\%) manifests that these data are suitable to represent the background and disturbances on the Sun.

The utility of observational data for the SSC is determined by the need for space weather forecast. Firstly, the forecast requires timely data, in other words, real-time or near-real-time data. Secondly, target data can be easily acquired and downloaded from their data sources. Thirdly, target data must be high quality for measuring the key objects and for forecasting space weather events. Fourthly, we use minimum target data to cover all the five key objects, and to make the SSC concisely. Based on the above four criteria, we find seven target data to construct the SSC. The parameters of the key objects, such as the net magnetic flux and unbalance of magnetic flux of the active region, are calculated and marked on the SSC for a quantitative chart.

\section{Examples of SSC}

In this section, we give two examples to explain the composition and application of SSC in details, which shows that the SSC can effectively analyze the overall situation of solar activities. Figure 1 shows an example of the SSC on 2012 March 6 . Figures 1 (a)-(c) are simultaneous SDO/AIA EUV 304, 171, and 131 $\AA$ images at $\sim$ 18:00 UT. Figure 1 (d) is an SDO/AIA EUV $193 \AA$ image at 18:00:07 UT, and it clearly shows a dark coronal hole that extended from the antarctic region to the equator of the Sun (see the arrow in the panel). Figure 1 (e) displays an SDO/HMI white-light image of the 


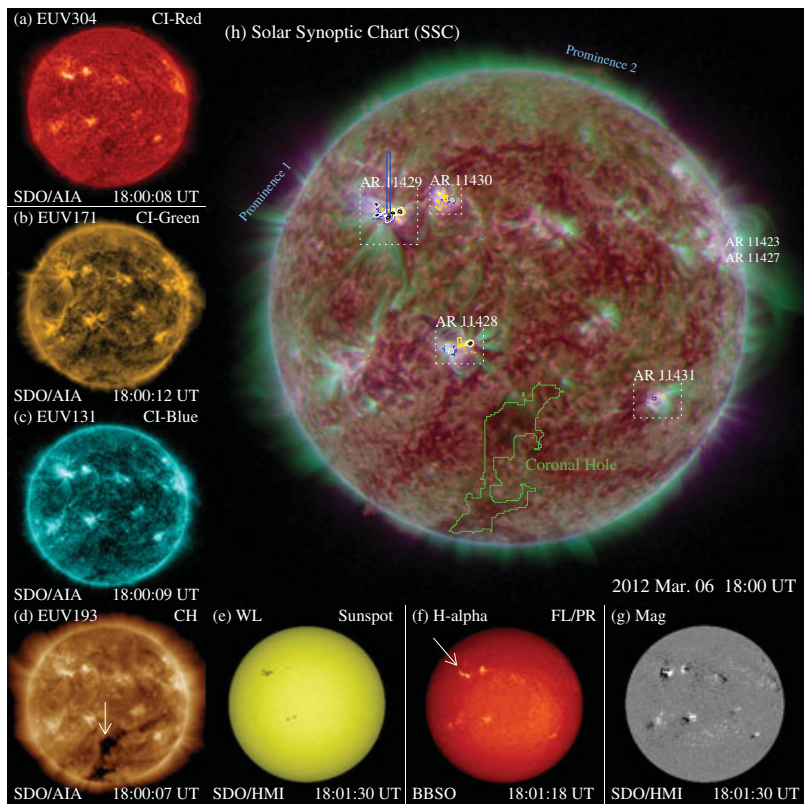

Figure 1. An example of solar synoptic chart (SSC) on 2012 March 6.

Sun at 18:01:30 UT and the dark spots on the yellow solar disk were sunspots. Figure 1 (f) is an $\mathrm{H} \alpha$ image at 18:01:18 UT from Big Bear Solar Observatory (BBSO). As indicated by the arrow in the panel, a filament was located next to an active region (AR 11429). Figure $1(\mathrm{~g})$ shows the distribution of longitudinal magnetic fields at 18:01:30 UT in a grey-scale magnetogram, and the black and white colors represent negative and positive polarities of magnetic fields, respectively.

As shown in Figure 1 (h), these seven nearly simultaneous data synthesize to one SSC, which contains a composite image overlaid with several contours and bars. Three colors, red, green, and blue, represent three SDO/AIA EUV images, 304, 171, and $131 \AA$ images, respectively, to make the composite image. Four active regions (AR 11428, AR 11429, AR 11430, and AR 11431) were on the disk, while the other two active regions (AR 11423 and AR 11427) were on the west limb of the Sun at 18:00 UT on 2012 March 6. Two prominences on solar limb are labeled in light blue. The colors, contours, and bars in SSC provide the information that cannot be analyzed from a single image. The black and white contours show the areas of umbra and penumbra of sunspots in the active regions. The yellow and blue contours represent strong positive and negative polarities of magnetic fields $( \pm 500 \mathrm{G})$ in the photosphere, respectively. The net magnetic flux is calculated and showed as a yellow or blue bar on each active region. The color of the bar depends on the polarity of the net magnetic flux in an active region, which is consistent with the colors of contours in the magnetogram. Besides these active regions, the boundary of the coronal hole on the southern hemisphere of the Sun is automatically identified and marked by green contours in the SSC.

AR 11429 and AR 11430 were two noticeable active regions on 2012 March 6. AR 11429 was bluer than AR 11430, which suggests that AR 11429 contained more hot plasma than its neighbor. As shown by the contours, AR 11429 had more complex photospheric structure than AR 11430. In its center, positive and negative magnetic structures shared the same penumbra, which indicates the presence of the $\delta$ sunspot. The polarity of leading (trailing) sunspots of AR 11429 was positive (negative), which is contrary to that of a 


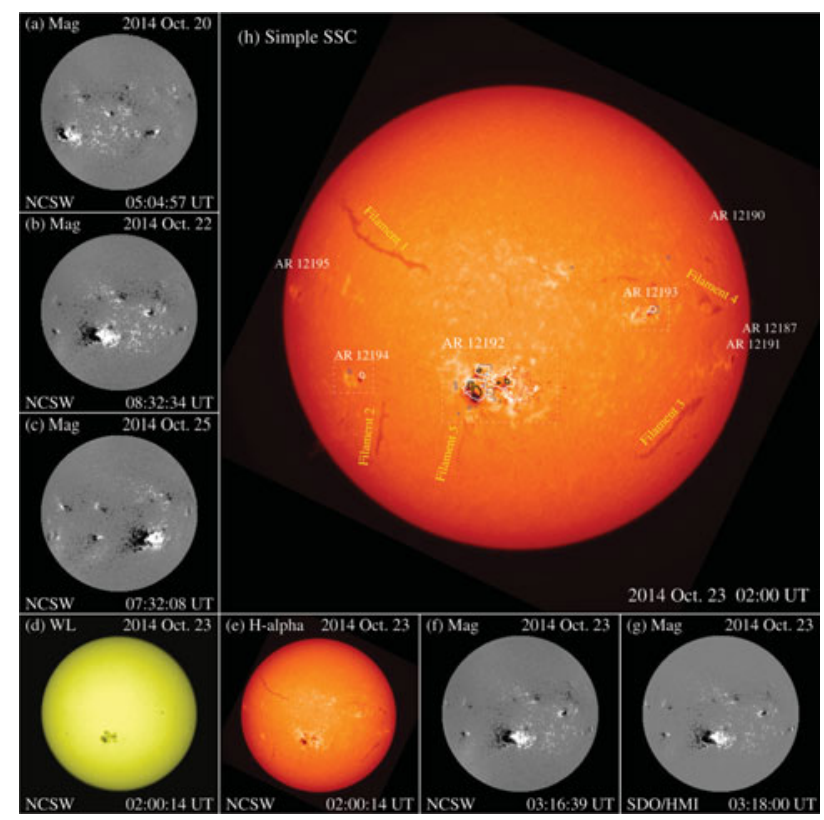

Figure 2. Simple version of SSC that made from the observations of NCSW on 2014 Oct. 23.

normal sunspot group on the solar northern hemisphere during Solar Cycle 24. The unbalances of magnetic flux were $-13 \%$ and $4.2 \%$ for AR 11429 and AR 11430. The net magnetic flux of AR 11429 and AR 11430 were $-3.4 \times 10^{20}$ and $2.7 \times 10^{19} \mathrm{Mx}$, and they are shown as a blue and a yellow bar on each active region, respectively. The high unbalances of magnetic flux of AR 11429 and its anomalous sunspot polarity suggest that it had greater possibility of eruptive activities than the other active regions did, which is consistent with the following observation. AR 11429 erupted two X-class flares on the next day. One X5.4 class flare started at 00:22 UT, 2012 March 7, meanwhile the filament near to AR 11429 erupted and produced a CME. When flares and CMEs erupt, the SSC can be used to trace their locations and evolutions in multiple height and temperature ranges.

Occasionally, the Internet service outage, the satellite eclipse, and the telescope maintenance or breakdown can cause a lack of external data sources, so we also design a simple version of SSC by using observations from NCSW to keep our forecast service running. Figures 2 (a)-(c) show longitudinal magnetograms of NCSW on 2014 October 20, 22, and 25, respectively. Figure 2(d) is a white-light image of NCSW at 02:00:14 UT, October 23. Figure 2(e) displays an $\mathrm{H} \alpha$ image of NCSW at the same time. Figures 2 (f) and (g) give a comparison between longitudinal magnetograms of NCSW and SDO/HMI. As illustrated by magnetic structures in different scales, the magnetogram of NCSW is consistent to that of $S D O / \mathrm{HMI}$. A small white dot appears in the middle of strong negative polarity in both magnetograms of NCSW and $S D O / \mathrm{HMI}$, and it is caused by the saturation effect. The $S D O / \mathrm{HMI}$ image has lower noise level than the NCSW image because $S D O$ is not disturbed by the Earth's atmosphere.

The simple SSC includes major information of solar activities despite the lack of EUV data. Figure 2(h) shows an example of simple SSC that contains seven active regions and five major filaments at 02:00 UT, 2014 October 23. Three active regions, AR 12192, AR 12193, and AR 12194, were on the solar disk, and the other four active regions were on the limbs. Among them AR 12192 had largest area and biggest net magnetic flux on 
October 23. The red and blue contours represent strong positive and negative polarities of magnetic fields in the photosphere, respectively. The black and white contours show the areas of umbra and penumbra of sunspots in active region. From the white-light and magnetic fields contours, we can see number, size, and polarity of sunspots of AR 12192 . The polarity of AR 12192 was normal and magnetic flux emergence was relatively slow. Five major filaments on solar disk are marked in yellow. Among them Filament 5 was close to AR 12192 and it might be triggered by the activities of AR 12192. The simple SSC indicates that the active region was very large, had a high possibility of flares, and had some possibilities of filament eruptions and coronal mass ejections. It is consistent with the SSC with EUV data and the following observation.

\section{Summary}

In this work, based on the investigation of key objects and the survey of observational data, we create the solar synoptic chart (SSC) for space weather forecast. The SSC includes a large number of information and its advantages are summarized as follows. 1) The SSC covers key objects of solar activities, including active regions, coronal holes, filaments/prominences, flares and CMEs. 2) The chart contains images from different heights and temperatures of solar atmosphere, and reveals magnetic structures from cool photosphere to hot corona. 3) The chart meets the need of space weather forecast because it is timely, quantitative, and comprehensive.

Furthermore, the chart can help in improving the public education for space weather because it is concise and easy to understand. The ultimate goal of the solar synoptic chart is to build a quantitative and interactive data system of the Sun for a more comprehensive understanding of space weather.

\section{Acknowledgements}

The data have been used by courtesies of BBSO and NASA/SDO. SDO is a mission for NASA's Living With a Star program. This work is supported by the National High-tech R\&D Program of China (2012AA121000) and the National Natural Science Foundation of China (41404136 and 40931056).

\section{References}

Hao, Q., Fang C., Chen P. F. 2013, Solar Physics, 286, 385

Henney, C. J., Harvey J. W. 2005, Astronomical Society of the Pacific Conference Series, 346, 261

Morgan, H., Byrne, J. P., \& Habbal, S. R. 2012, ApJ, 752, 144

Qu, M., Shih, F. Y., Jing, J., \& Wang, H. 2003, Solar Physics, 217, 157

Schwenn, R. 2006, Living Reviews in Solar Physics, 3, 2

Song, Q., Zhang, J., Yang, S. H., \& Liu, Y. 2013, Research in Astronomy and Astrophysics, 13, 226

Stys, J. E., Ireland, J., \& Müller, D. 2015, AAS/AGU Triennial Earth-Sun Summit, 1, 40304

Verbeeck, C., Higgins, P. A., Colak, T., Watson, F. T., Delouille, V., Mampaey, B., \& Qahwaji, R. 2013, Solar Physics, 283, 67 